\title{
Quantum Metametaphysics
}

\author{
Alessandro Torza
}

\begin{abstract}
Say that metaphysical indeterminacy occurs just when there is a fact such that neither it nor its negation obtains. The aim of this work is to shed light on the issue of whether orthodox quantum mechanics provides any evidence of metaphysical indeterminacy by discussing the logical, semantic, and broadly methodological presuppositions of the debate. I argue that the dispute amounts to a verbal disagreement between classical and quantum logicians, given Eli Hirsch's account of substantivity; but that it need not be so if Ted Sider's naturalnessbased account of substantivity is adopted instead. Given the latter approach, can anything be said in order to tip the balance of the dispute either way? Some prima facie reasonable constraints on naturalness entail that the classicist is right, and the quantum world is therefore determinate. Nevertheless, there are reasons for weakening those constraints, to the effect that the dispute remains very much open. Finally, I discuss alternative accounts of metaphysical indeterminacy, and argue that they are unsuitable for framing the quantum indeterminacy debate.

Keywords: disagreement; metaphysical indeterminacy; quantum indeterminacy; quantum logic; nonclassical logic; natural properties.
\end{abstract}

\section{Introduction}

A significant shift in the recent literature about indeterminacy concerns the idea that the phenomenon of indeterminacy could originate not only from the way we represent the world, but also from the world itself. Representational indeterminacy arises when the way we describe reality, linguistically or otherwise, is somehow defective. ${ }^{1}$ Worldly, aka metaphysical indeterminacy occurs just when it is indeterminate what reality is like, irrespectively of

\footnotetext{
${ }^{1}$ Representational indeterminacy is usually construed along semantic or epistemic lines. The locus classicus for a semantic treatment of representational indeterminacy is Fine [20]. The alternative, epistemic account dates back to Williamson [47].
} 
how it is represented. ${ }^{2}$ In this work I will be concerned with indeterminacy of the worldly variety.

Since quantum mechanics is arguably the most successful scientific theory at our disposal, it is only reasonable that close attention should be paid to any evidence which that theory may have to offer in favor or against metaphysical indeterminacy. It is a well known fact that, according to Heisenberg's uncertainty principle, it is impossible to assign a sharp value to each experimental property, aka observable of a system at the same time. There are sets of observables-like position and momentum, or spin components along different directions - such that the sharper one of the observables, the less sharp the remaining ones in the same set.

A growing number of philosophers have defended, or at least entertained the idea that the orthodox interpretation of quantum mechanics provides us with reasons to believe that reality is indeterminate (Bokulich [7], Calosi and Wilson [9] [10], Darby [14], Darby and Pickup [15], Lewis [29, ch. 4], Lowe [30] [31], Skow [39], Torza [40], Williams [45]). Nevertheless, a cursory survey of the literature will show that not only we have not reached a consensus on the issue, but it has also been argued that interpreting quantum mechanics as supporting metaphysical indeterminacy is hasty, if not misguided (Noonan [35], Glick [21]). ${ }^{3}$

In this work I will not take sides on the first-order debate as to whether the world is, at the quantum level, indeterminate. Instead, I will attempt to shed light on that debate by addressing a number of (mostly) higher-order questions, namely:

- How is the debate about quantum metaphysical indeterminacy best understood in light of the underlying logic?

- Is the debate substantive, or is it merely verbal?

- If the debate is substantive, are there any higher-order considerations that could tip the balance of the first-order debate either way?

I will start off by taking a first stab at a characterization of metaphysical indeterminacy (section 2). Next comes a discussion of the main logical frameworks that are typically associated with quantum theory, namely classical logic and quantum logic. I will argue that metaphysical indeterminacy

\footnotetext{
${ }^{2}$ Among the proposed accounts of metaphysical indeterminacy are Akiba [1], Barnes and Williams [5], Torza [40], Williams [46], Williamson [48], Wilson [50].

${ }^{3}$ For an overview of the state of the art on quantum indeterminacy, see Calosi and Mariani [8].
} 
will arise if we interpret quantum mechanics on a quantum logical space, and it won't if we interpret it on a classical logical space. Since both options are admissible, a stalemate is reached (section 3). As it turns out, the stalemate is rooted in the fact that the disagreement between the two parties - the advocates of classical vs quantum logic - regarding quantum indeterminacy is verbal (section 4 ). Such a deflationary moral can be avoided by adopting a stricter characterization of metaphysical indeterminacy, as well as of verbal disagreement. In particular, if we embrace a metaphysics of naturalness, the quantum MI debate need not be verbal; and it is substantive provided that either classical or quantum negation is natural (section 5). Some prima facie reasonable constraints on naturalness entail that quantum negation cannot be natural, which lends support to the no-indeterminacy view of quantum mechanics. However, I will make a case for adopting less demanding constraints on naturalness, which do not tip the balance of the debate either way. On such grounds, the dispute on quantum MI remains very much open (section 6). Finally, I will explain why I have not adopted one of the main characterizations of MI in the market - the precisificational account of Elizabeth Barnes and Robert Williams, or the determinable-based account of Jessica Wilson. I explain that the former is unable to model MI of the quantum variety; whereas the latter rules out quantum logic on dubious metaphysical grounds (section 7).

\section{Metaphysical indeterminacy}

I am going to assume two constraints on any viable characterization of MI. First of all, MI should be defined solely by reference to worldly items, whether concrete or abstract (individuals, properties, facts, operations, etc), as opposed to representational items (names, predicates, sentences, operators, etc). MI being a thesis about the world, a roundabout approach appealing to representational machinery is best avoided. This assumption is meant to guarantee that MI is indeed characterized as a worldly, rather than a representational phenomenon.

There are two reasons for avoiding a characterization of MI in terms of representational items. One reason is that such a characterization might overgenerate cases of MI due to potential defectiveness of the representational items being employed. For instance, if MI is characterized by appealing to the representational properties of a language $\mathcal{L}$, we will inevitably end up with false positives of MI if some term of $\mathcal{L}$ is semantically vague or irreferential. Although we might be able to rule out the false positives 
by imposing strict conditions on $\mathcal{L}$, the strategy comes at a price, in that substantive semantic assumptions will have to be made. ${ }^{4}$ Another reason not to appeal to representational items in characterizing MI is that, since any representational medium (such as a language) suffers from some expressive limitation or other, there is always risk that such a characterization will undergenerate cases of MI.

The second constraint is that, other things being equal, we should prefer a characterization that makes metaphysical indeterminacy structurally similar to its representational counterpart, in such a way that the two phenomena will share some important feature in virtue of which they can both be said to be notions of indeterminacy. Because this is merely a ceteris paribus condition, it does not rule out in principle the viability of a gerrymandered notion of indeterminacy, whereby the representational and metaphysical cases share no nontrivial features. However, unless we have good reasons to believe that our use of the term 'indeterminacy' for referring to both phenomena is nothing more than an unfortunate ambiguity, I take nondisjunctive conceptions to be preferable.

With that being said, here is a first-pass characterization to the effect that MI arises just when there is a gap in logical space. (A finer characterization is put forward in section 5.2.)

IND. Metaphysical indeterminacy is said to occur if there is a fact such that neither it nor its negation obtains.

A few observations are in order. First of all, I am using 'fact' as synonymous with 'state of affairs', in such a way that facts are the nonrepresentational correlates of propositions (cf. Williamson [48, p. 699]). On this view, the fact that Marie is Canadian exists whether Marie is or is not Canadian; and the fact that Marie is Canadian obtains if and only if Marie is Canadian. In the following discussion I will be implement a coarse-grained construal of facts, to the effect that facts $F, G$ are identical if and only if it is necessarily the case that $F$ obtains if and only if $G$ obtains (relative to a given logical space)..$^{5}$

\footnotetext{
${ }^{4}$ The issue is illustrated by the characterization of MI put forward in Torza [40], according to which MI occurs just when (roughly) there is a sentence in a semantically nondefective language $\mathcal{L}$ which is neither true nor false. As Torza points out, his characterization runs into false positives of MI unless one assumes (i) a Russellian account of definite descriptions, and (ii) either a negative or a positive free semantics for $\mathcal{L}$.

${ }^{5}$ Although facts can be taken to be metaphysically fundamental (Rayo [37], Turner [42]), I will refrain from taking sides on such matters, which are orthogonal to the present concerns.
} 
Second, IND meets the constraint that only worldly items should be employed in defining MI. Indeed, three kinds of items are mentioned in the definiens: fact, negation, and obtaining. Each of them contributes to the objective, mind-independent structure of logical space, defined as a collection of facts together with whatever structure is required for the interpretation of our logical vocabulary. ${ }^{6}$ Insofar as the structure of logical space is worldly, so are the entities mentioned in IND.

The above definition of MI also meets the desideratum that metaphysical and representational indeterminacy should be structurally similar. In particular, if 'sentence', 'negation (operator)' and 'is true' are substituted in IND for 'fact', 'negation (operation)' and 'obtains', respectively, we end up with a characterization of representational indeterminacy which is shared by a number of standard theories, among them supervaluationism. A relevant example of representational indeterminacy is provided by the sentence 'Laura is tall', which will be neither true nor false if 'tall' is a vague predicate having Laura as a borderline case. Mutatis mutandis, if 'sentence', 'negation (operator)' and 'is knowable to be true' are substituted in IND for 'fact', 'negation (operation)' and 'obtains', respectively, we end up with a characterization of representational indeterminacy along epistemicist lines.

I do not expect the above characterization of MI to be uncontroversial, mainly because it differs substantially from the two main options on the market: the precisificational account of Elizabeth Barnes and Robert Williams, and the determinable-based account of Jessica Wilson. I will return to those alternatives in section 7 , where it is argued that they both run into important problems when it comes to modeling quantum indeterminacy.

\section{Logical space}

\subsection{Classical logical space}

The objective, mind-independent space of absolutely all possibilities is known as logical space. This paper deals with logical space in a restricted sense, namely as the mind-independent space of all nomological possibilities (i.e., possibilities compatible with the relevant laws of nature). Whenever I am considering a particular physical system, by 'logical space' I will mean the space of what is nomologically possible for that system.

\footnotetext{
${ }^{6}$ Note that in IND by 'negation' I mean an objective, worldly operation in logical space mapping facts to facts, and not a linguistic, representational operator mapping formulas to formulas.
} 
The definition of a logical space can proceed from different choices of basic elements: individuals, properties, worlds etc. I am going to assume that any logical space meets the following conditions: (i) it features a class of facts; (ii) it is endowed with a relational property of obtaining-at; and (iii) it is closed under a number of logical operations. Once (i)-(iii) are in place, logical spaces can be employed in order to define a model theory for sentential logic in the standard way.

A classical logical space is a structure $\mathcal{C}=\left\langle C\right.$, @, $\left.\mathrm{T}_{C},-{ }_{C}, \Pi_{C},\right\rangle$ such that:

1. $C$ is a set of entities playing the role of possible maximal states, aka worlds. Among them is a distinguished maximal state @, the actual world.

2. Facts are sets of worlds. The total set $C$ is the necessary fact; the empty set is the impossible fact.

3. A fact $P$ is said to obtain at world $w$ (in symbols, $\mathrm{T}_{C}(P, w)$ ) if $w \in P$; it is said to obtain simpliciter if $\mathrm{T}_{C}(P, @)$.

4. Logical operations are identified with the standard set-theoretic operations over $C$ : factual negation $\left(-{ }_{C} P\right)$ is complementation, and factual conjunction $\left(P \sqcap_{C} Q\right)$ is intersection.

The factual disjunction $P \sqcup_{C} Q$ is defined as $-_{C}\left(-{ }_{C} P \sqcap_{C}-{ }_{C} Q\right)$, which is set-theoretic union.

Classical logical space thus defined meets the aforementioned conditions (i)-(iii). Classical (sentential) logic is the logic obtained by interpreting a sentential language on a classical logical space, and identifying logical truth with truth in all classical logical spaces. ${ }^{7}$

It is straightforward to see that MI cannot occur in classical logical space as defined above. Indeed, for any fact $P$, either $@ \in P$ or $@ \notin P$. Therefore, either @ $\in P$ or $@ \in{ }_{C} P$; which is to say, either $P$ or its classical negation obtains.

\subsection{Quantum logical space}

The orthodox interpretation of quantum mechanics is typically associated with three principles: the Schrödinger equation, which defines the dynamics

\footnotetext{
${ }^{7}$ It goes without saying that this way of modeling logical space does not commit one to full-blown modal realism, or to any substantive thesis typically associated with Lewisean metaphysics, since in the present context 'world' is being used merely as an umbrella term compatible with alternative views about the foundations of modality.
} 
of a system; the Born rule, which specifies the probabilities of quantum experiments; and the eingestate-eigenvalue link, which provides necessary and sufficient conditions for ascribing a physical property to a system given its state. Instead of stating those principles here, I will do so when required. The truth of the orthodox interpretation will be assumed throughout. ${ }^{8}$

The transition to the realm of quantum physics makes the task of determining the structure of logical space more challenging. The standard way of representing a space of possible states of a quantum system appeals to a particular kind of structure known as a Hilbert space. ${ }^{9}$ What matters for present purpose is that a Hilbert space $\mathcal{H}$ is a vector space, and is therefore closed under linear combination of vectors; and that the state of a system is represented by a unitary vector. One ubiquitous feature of quantum systems is the phenomenon of superposition, which is understood via linear combination. In the language of Hilbert spaces: if $\left|\psi_{1}\right\rangle,\left|\psi_{2}\right\rangle$ are states in $\mathcal{H}$, then for any real or complex scalars $\alpha, \beta, \alpha\left|\psi_{1}\right\rangle+\beta\left|\psi_{2}\right\rangle$ is also a state in $\mathcal{H}$, and is said to be a superposition of $\left|\psi_{1}\right\rangle$ and $\left|\psi_{2}\right\rangle$.

One might think that, by analogy with the classical case, we already have all we need in order to specify a quantum logical space: quantum states are unitary vectors in a Hilbert space; facts are sets of vectors; obtaining-at is defined by set-membership; and logical operations are the standard settheoretic ones. If that is the case, MI is also ruled out in the quantum world. This conclusion, however, runs afoul of some reasonable constraints.

A physically meaningful, aka experimental fact is a fact consisting of a system having some physical property. The class of physical properties is the smallest class comprising both determinate physical quantities and logical compounds thereof. An example of a fact involving some determinate physical quantity is an electron's having spin up along a specified direction. An example of a fact involving some logical compound of determinate physical quantities is an electron's having either spin up or spin down along a specified direction.

\footnotetext{
${ }^{8}$ Although the orthodox interpretation is often paired with an instrumentalist attitude toward quantum theory, it seems to me that a realist reading is more natural. Indeed, the eigenstate-eigenvalue link, which establishes a connection between formalism and physical properties, is incompatible with some of the main available realist interpretations. (Similar considerations carry over to the Criterion of Reality discussed later in this section.) Cf. Bokulich [7, p. 460 fn. 14] for a defense of a realist attitude toward the orthodox interpretation.

${ }^{9} \mathrm{~A}$ Hilbert space is defined as a complete vector space with an inner product. Hilbert spaces can be finitely or infinitely dimensional. Although I will restrict my attention to the finite case, the metaphysical moral discussed here carries over to infinitely dimensional spaces.
} 
If $S$ is an experimental fact, let $\operatorname{Pr}(S,|\psi\rangle) \in[0,1]$ be the probability that $S$ obtains upon measurement provided that the state of the system is $|\psi\rangle$. The Criterion of Reality, which was first stated by Einstein, Podolski and Rosen [18, p. 777] within the context of the debate concerning the descriptive completeness of quantum mechanics, says that 'if, without in any way disturbing a system, we can predict with certainty (i.e., with probability equal to unity) the value of a physical quantity, then there exists an element of physical reality corresponding to this physical quantity.' This principle 'provides us with a sufficient condition for an electron to have a spin property, namely if we can predict with probability 1 the spin we will obtain on measurement' (Lewis [29, p. 28]).

As stated, the Criterion of Reality will apply to all facts consisting of a system's having some determinate physical quantity. But, I claim, it should also hold for logically complex facts, if it holds at all. For example, we want to say that if the probability that an electron is found in a given region $R$ is 1 , then the electron is located in that region. But if $r_{0}, r_{1}, \ldots, r_{n}$ is a partition of $R$, being located in $R$ is tantamount to the complex property of being located in $r_{0}$ or being located in $r_{1}$ or... or being located in $r_{n}$. So, we will also want to say that if the probability that an electron is found in region $r_{0}$ or it is found in region $r_{1}$ or... or it is found in region $r_{n}$ is 1 then the electron is located in $r_{0}$ or it is located in $r_{1}$ or... or it is located in $r_{n}$.

The above point holds with a proviso. One might worry that being located in a region cannot amount to being located in one of its subregions. For the partition can be defined in such a way that the individual cells $r_{0}, r_{1}, \ldots, r_{n}$ are arbitrarily small, so that being located in any particular cell $r_{i}$ asymptotically amounts to having definite position - which contradicts the fact that a quantum particle can be located in a region $R$ while lacking definite position. However, the worry rests on a compositional account of logical operations, whereby a disjunctive fact obtains just in case one of the disjuncts obtains. Thus, extending the Criterion of Reality to complex facts is not in contradiction with the empirical evidence, as long as compositionality is abandoned. (I will return to the issue of compositionality at the end of this subsection.)

Now, if we assume that the Criterion of Reality indeed holds for experimental facts of arbitrary logical complexity, we can state it as follows, for $S$ an experimental fact:

CR. If $\operatorname{Pr}(S,|\psi\rangle)=1$ then $S$ obtains at $|\psi\rangle$.

Recently, Maudlin $[32$, p. 6] has gone so far as to claim that the Criterion 
of Reality is analytic. ${ }^{10}$ Be that as it may, since the Criterion of Reality is widely regarded as a minimal tenet of realism about quantum mechanics, it will not be unreasonable to assume it, provided that quantum mechanics is a reliable guide to the structure of reality. ${ }^{11}$

What makes CR relevant to the present discussion is that it entails the following condition:

CLOSURE. If $|\psi\rangle$ is a superposition of $\left|\psi_{1}\right\rangle,\left|\psi_{2}\right\rangle, \ldots\left|\psi_{n}\right\rangle$, and $S$ obtains at each $\left|\psi_{i}\right\rangle_{i \leq n}$, then $S$ obtains at $|\psi\rangle .^{12}$

In other words, an experimental fact obtaining at each vector component of a state $|\psi\rangle$ will also obtain at $|\psi\rangle$. An immediate corollary is that the experimental facts of quantum mechanics are closed under linear combination (Birkhoff and von Neumann [6, p. 826]).

We can now see that the initial sketch of quantum logical space went too fast, in that it assumed that any set of vectors in a Hilbert space can be identified with some physically meaningful fact, whereas only a special subclass of them can, namely the closed ones. Because of that, complementation cannot play the role of factual negation, since in general it does not map closed sets to closed sets. For example, in a two-dimensional $z$-spin Hilbert space for a single spin- $\frac{1}{2}$ particle, the set-theoretic complement of the fact that the particle is $z$-spin up is not a necessary fact and contains an orthonormal basis, and is therefore not closed.

The above remarks motivate the introduction of quantum logical space (Birkhoff and von Neumann [6]) which is a structure $\left.\mathcal{Q}=\left\langle\mathcal{H}, \mid \psi_{@}\right\rangle, \mathrm{T}_{Q},-{ }_{Q}, \sqcap_{Q}\right\rangle$ such that:

1. $\mathcal{H}$ is a Hilbert space, where unitary vectors are possible maximal states. Among them is an actual state $\left|\psi_{@}\right\rangle$.

2. Facts are sets of vectors in $\mathcal{H}$ closed under linear combination. The total set is the necessary fact, whereas the empty set is the impossible fact.

\footnotetext{
${ }^{10}$ But see Glick and Boge [21] for a dissenting voice.

${ }^{11}$ Could one accept the Criterion of Reality while denying $\mathrm{CR}$ on the grounds that the 'element of physical reality' need not be the predicted property itself? That is certainly an admissible view, but not the intended one in the EPR paper: "in the state given by $\left[\psi=e^{(2 \pi i / h)} p_{0} x\right]$, the momentum has certainly the value $p_{0}$. It thus has meaning to say that the momentum of the particle in the state given by [that equation] is real" (Einstein, Podolski and Rosen [18, p. 778], my emphasis).

${ }^{12}$ Proof: The Born rule, which specifies the probabilities of quantum experiments, tells us that if $|\psi\rangle=\alpha_{1}\left|\psi_{1}\right\rangle+\ldots+\alpha_{n}\left|\psi_{n}\right\rangle$ and $S$ obtains at $\left|\psi_{i}\right\rangle$ then $\operatorname{Pr}(S,|\psi\rangle)=\left|\alpha_{i}\right|^{2}$, where $\left|\alpha_{1}\right|^{2}+\ldots+\left|\alpha_{n}\right|^{2}=1$. Since $S$ is assumed to obtain at $\left|\psi_{i}\right\rangle$, for each $i \leq n$, then $\operatorname{Pr}(S,|\psi\rangle)=1$. By CR, we can conclude that $S$ obtains at $|\psi\rangle$.
} 
3. A fact $S$ is said to obtain at $|\psi\rangle$ (in symbols, $\mathrm{T}_{Q}(S,|\psi\rangle)$ ) if $|\psi\rangle \in S$; it is said to obtain simpliciter if $\mathrm{T}_{Q}\left(S,\left|\psi_{@}\right\rangle\right)$.

4. The following are logical operations on $\mathcal{Q}$ :

(a) factual negation $\left(-{ }_{Q} S\right)$ is orthocomplementation. The orthocomplement of a fact $S$ is the set of vectors that are orthogonal to each vector in $S$.

(b) factual conjunction $\left(S \sqcap_{Q} T\right)$ is intersection.

The factual disjunction $S \sqcup_{Q} T$ is defined as $-{ }_{Q}\left(-{ }_{Q} S \sqcap_{Q}-{ }_{C} T\right)$, which corresponds to span. The span of $S, T$ is the closure of the set-theoretic union $S \sqcup_{C} T$ under linear combination.

Quantum logical space thus defined meets conditions (i)-(iii) as stated in section 3.1. Quantum (sentential) logic is the logic obtained by interpreting a sentential language on quantum logical space, and identifying logical truth with truth in all quantum logical spaces. ${ }^{13}$

One can now see that MI will arise in quantum logical space, for if $\left|\psi_{@}\right\rangle$ is neither a member of $S$ nor a member of its orthocomplement, then neither $S$ nor $-{ }_{Q} S$ obtains. For example, consider a one-electron system where $S$ is the fact that the electron $e$ is spin up along the $z$ direction (i.e., $\left.S=\left\{\alpha\left|\uparrow_{z}\right\rangle_{e}: \alpha \in \mathbb{C}\right\}\right)$. The orthocomplement $-{ }_{Q} S$ is none other than the fact that $e$ is spin down along the $z$ direction (i.e., ${ }_{Q} S=\left\{\alpha\left|\downarrow_{z}\right\rangle_{e}: \alpha \in \mathbb{C}\right\}$ ). If $\left|\psi_{@}\right\rangle=\frac{1}{\sqrt{2}}\left(\left|\uparrow_{z}\right\rangle_{e}+\left|\downarrow_{z}\right\rangle_{e}\right)$, then $\left|\psi_{@}\right\rangle \notin S$ and $\left|\psi_{@}\right\rangle \notin-{ }_{Q} S$, which means that it is metaphysically indeterminate whether $e$ is spin up or spin down along the $z$ direction.

There is one last issue that was left pending. The argument I offered in support of CLOSURE relies on a strong reading of the Criterion of Reality, captured by $\mathrm{CR}$, which applies not only to facts involving a determinate property, but also to facts involving logical complex properties. My justification for the strong reading hinged on a rejection of compositionality. That move would now look suspicious, however, if CR had been wielded to introduce a compositional logical space. The worry can be put to rest, insofar as

\footnotetext{
${ }^{13}$ Putnam [36] argued that quantum logic is the one true logic; and that the paradoxes of quantum mechanics, especially the measurement problem, are solved by adopting quantum logic (cf. Bacciagaluppi [3]). What I have said so far does not entail either of Putnam's theses. Indeed, quantum logic's being the correct logic for modeling reasoning about quantum systems is compatible with both quantum logic's not being the one true logic, and with the measurement problem's not being solvable by appealing to quantum logic. Nevertheless, the question whether classical or quantum logic is the the one true logic is relevant to the present debate, and I will return to it in section 5.
} 
quantum logic is clearly noncompositional. For example, if our one-electron system is in a superposition of $z$-spin states, the fact that $e$ is either $z$-spin up or $z$-spin down (i.e., $S \sqcup_{Q}-{ }_{Q} S$ ) will obtain, even though neither disjunct obtains. The reason is that the span of the set $\left\{S,-{ }_{Q} S\right\}$ coincides with the necessary fact in the quantum logical space for the system at hand.

\subsection{Stalemate}

We have seen that MI arises in quantum mechanics, if this is interpreted on a quantum logical space. The logical classicist will not go down so easily, however. The idea that our best theories are determinate is deeply ingrained in the history of science and especially physics, as Eddy Chen reminds us:

"All the paradigm cases of candidate fundamental laws of nature are not only simple and universal, but also exact, in the sense that, for every class of worlds (or class of solutions), fundamental laws either determinately apply or determinately fail. [...] The kind of mathematics we are used to, built from a set-theoretic foundation, does not lend itself naturally to model the genuine fuzziness and indeterminacy of vagueness" (Chen [12]).

In a similar vein, here is an argument to the effect that quantum mechanics does not support logical revisionism in general, and quantum logic in particular:

Quantum mechanics is formulated in classical mathematics, which obeys classical logic. Since the intended semantics for classical logic is bivalent, there is a straightforward way of interpreting quantum mechanics in such a way that every quantum fact is such that either it or its negation obtains. Hence, not only quantum mechanics does not force the adoption of a quantum logical space upon us, but its default interpretation involves a classical logical space.

The gist of the classicist's rejoinder is that since the structure of a Hilbert space boils down to classical linear algebra which, as any run-of-the-mill mathematical theory, is interpretable on a classical logical space, the transition from classical to quantum physics does not require a transition from classical to quantum logic spaces, and so provides no support for MI. ${ }^{14}$

\footnotetext{
${ }^{14}$ It is worth noting that MI is prima facie compatible with conservatism about logic. Indeed, the account of MI due to Wilson [50] is formulated against the backdrop of a classical and bivalent logic (but see sec. 7 for criticism). Likewise, the model theory of MI developed
} 
By way of example, consider an equation of the form $\hat{O}|\psi\rangle=\lambda|\psi\rangle$, where $\hat{O}$ is a linear operator associated with the observable property $O$. Both the classical and the quantum logician will agree that the equation expresses the fact that the system has property $O$ with value $\lambda .{ }^{15}$ In a Hilbert space, that fact is identified with the set $S$ of unitary vectors differing from $|\psi\rangle$ by a scalar. According to the classicist, however, the (classically) negated sentence $\hat{O}|\psi\rangle \neq \lambda|\psi\rangle$ also expresses a fact, corresponding to the set-theoretic complement of $S$. The quantum logician, on the other hand, will insist that $\hat{O}|\psi\rangle \neq \lambda|\psi\rangle$ as uttered by the classicist has no experimental meaning, since it does not pick out a closed set, and therefore fails to express a physically meaningful fact about the system at hand.

We seem to have reached a stalemate. Quantum logicians will address the opponents with the following speech:

You think you can avoid MI by introducing redundant structure, namely by postulating semantic values for sentences governed by classical connectives. But those extra 'facts' of yours have no physical content, and should therefore be purged from the logical space of quantum physics.

Classicists, on the other hand, will likely stick to their guns and reply:

You identify facts with closed sets in a Hilbert space. But closed sets only constitute a subclass of all facts statable in the language of quantum mechanics, viz. the ones associated with some possible experiment. MI results from unduly restricting the class of facts to the experimental facts.

by Barnes and Williams [5] validates all classical schemas and inference rules, and makes every (semantically precise) statement either true or false. However, the Barnes-Williams strategy is precisificational and, as I explain in sec. 7, precisificational approaches have proven unsuitable to modeling quantum indeterminacy. Moreover, the Barnes-Williams model recovers bivalence by driving a wedge between truth and determinacy, insofar as it does not validate the inference from $p$ to $D p$ (where $D$ is an object-language metaphysical determinacy operator), while failing to explain what it means for the world to contain a truthmaker for the former but not the latter. (Cameron [11] suggests that it amounts to $p$ 's truthmaker to exist but not definitely so, which merely pushes the bump under the rug.)

${ }^{15}$ This inference assumes the eigenstate-eigenvalue link: a quantum system has property $O$ with value $\lambda$ iff the quantum state of the system is in an eigenstate of the associated operator $\hat{O}$ with eigenvalue $\lambda$. The eigenstate-eigenvalue link is a tenet of the orthodox interpretation of quantum mechanics (sec. 3.2). It is worth noting however that David Wallace has argued that the eigenstate-eigenvalue link has never belonged to the orthodox interpretation (Wallace [43]), and that it is false (Wallace [44]). See Gilton [24] for a defense of the eigenstate-eigenvalue link as a traditional component of the orthodox interpretation. Whether the link is indeed correct is a question that lies beyond the scope of this work. 
Since one party affirms and the other denies that quantum mechanics leads to MI, one's knee jerk reaction might be to search for faulty premises on either side of the debate. In the next section I will argue instead that the stalemate may be an illusion, insofar as the dispute between classical and quantum logician turns out to be merely verbal, given a standard understanding of what counts as a verbal dispute.

\section{Substantivity}

In the present section, I am going to offer necessary and sufficient conditions for a debate to be verbal (nonsubstantive) by drawing on Hirsch [22] [23]. Once the relevant concepts are in place, I will employ them in order to assess the substantivity of the debate on quantum MI.

Let $\mathcal{L}_{A}, \mathcal{L}_{B}$ be interpreted languages spoken by $A, B$, respectively, and let $p$ be a sentence formulated in the common vocabulary. The two parties are said to be having a verbal disagreement about $p$ 's truth just in case:

1. there is a two-way paraphrase between $\mathcal{L}_{A}$ and $\mathcal{L}_{B}$;

2. $p$ is true-in- $\mathcal{L}_{A}$ iff it is not true-in- $\mathcal{L}_{B}$.

A couple of observations are in order. First, $p$ 's being true-in- $\mathcal{L}_{A}$ means that the semantics of $\mathcal{L}_{A}$ maps $p$ to an actually obtaining fact. Second, to say that $\mathcal{L}_{A}$ can be paraphrased into $\mathcal{L}_{B}$ means that there is a mapping $\mathbf{f}$ of $\mathcal{L}_{A}$-sentences to $\mathcal{L}_{B}$-sentences that preserves coarse-grained content, i.e., such that $p$ expresses-in- $\mathcal{L}_{A}$ fact $S$ only if $\mathbf{f}(p)$ expresses-in- $\mathcal{L}_{B}$ fact $S$. The existence of a two-way paraphrase is crucial to capturing the notion that the parties are not disagreeing on any worldly matters, even though they may describe things differently.

I am going to start by arguing that the above condition (1) is satisfied by $\mathcal{L}_{C}, \mathcal{L}_{Q}$, where $C, Q$ are the classical and quantum logician, respectively. The crux of the argument I am about to offer is that, although $C$ and $Q$ disagree about the truth value of sentences concerning the quantum world because of the different interpretations they assign to the negation symbol, the disagreement can be reconciled by appealing to further expressive resources. In particular, the classicist can charitably interpret the quantum logician in probabilistic terms, whereas the quantum logician can charitably interpret the classicist by semantic ascent to a classical metalanguage.

Categorical sentences are sentences about a physical system that do not involve intensional operators, truth predicates, (in)determinacy operators, or anything of the sort. Atomic categorical sentences of quantum mechanics 
are sentences that ascribe determinate physical quantities to a system-e.g., ' $t_{1}, \ldots, t_{n}$ are in such and such position', or ' $t_{1}, \ldots, t_{n}$ have such and such spin'. Under informationally ideal conditions there is going to be no disagreement between a classicist and a quantum logician regarding the truth values of those sentences. Compound categorical sentences are recursively defined by the application of sentential operators $(\wedge, \neg)$ to categorical sentences, and ascribe logical compounds of determinate physical quantities to a system. ${ }^{16}$ Since the categorical fragments of $\mathcal{L}_{C}$ and $\mathcal{L}_{Q}$ only differ by the way the negation symbol is interpreted, the relevant disagreement between the two parties will only arise in the case of compound categorical sentences.

In order to show that $C$ and $Q$ disagree verbally, we need to provide a paraphrase from the categorical fragment of $\mathcal{L}_{C}$ into $\mathcal{L}_{Q}$, and a paraphrase from the categorical fragment of $\mathcal{L}_{Q}$ into $\mathcal{L}_{C}$. Let us start with the latter. Before giving a precise definition, it will help to state the main idea with an example. When the quantum logician asserts an atomic categorical sentence of $\mathcal{L}_{Q}$, say ' $e$ is $z$-spin up', the classicist can charitably interpret it as picking out the fact expressed-in- $\mathcal{L}_{C}$ by 'the probability that $e$ is found to be up on a $z$-spin measurement is $1^{\prime} .{ }^{17}$ And when the quantum logician asserts ' $e$ is not $z$-spin up', the classicist can charitably interpret it as picking out the fact expressed-in- $\mathcal{L}_{C}$ by 'the probability that $e$ is found to be up on a $z$-spin measurement is 0 '.

Now, for any speaker $A$ and sentence $P$ of $\mathcal{L}_{A}$, let the fact $[P]_{A}$ be the interpretation-in- $\mathcal{L}_{A}$ of $P$. A paraphrase $\mathbf{f}$ from the categorical fragment of $\mathcal{L}_{Q}$ into $\mathcal{L}_{C}$ is recursively defined as follows:

$\left(\mathrm{At}_{Q}\right)$ If $p$ is an atomic categorical sentence of $\mathcal{L}_{Q}$, then $\mathbf{f}(p)$ is $\operatorname{Pr}\left([p]_{C},\left|\psi_{@}\right\rangle\right)$ $=1$.

$(\neg Q)$ If $P$ is a categorical sentence of $\mathcal{L}_{Q}$, then $\mathbf{f}(\neg P)$ is $\operatorname{Pr}\left([\mathbf{f}(P)]_{C},\left|\psi_{@}\right\rangle\right)=$ 0 .

$\left(\wedge_{Q}\right)$ If $S, P$ are categorical sentences of $\mathcal{L}_{Q}$, then $\mathbf{f}(S \wedge P)$ is $\operatorname{Pr}\left([\mathbf{f}(S)]_{C},\left|\psi_{@}\right\rangle\right)$ $=1 \wedge \operatorname{Pr}\left([\mathbf{f}(P)]_{C},\left|\psi_{@}\right\rangle\right)=1$.

\footnotetext{
${ }^{16} \mathrm{I}$ will be assuming that sentences such as ' $t$ is in region $R$ ' or ' $t$ has $z$-spin' are covert compound categorical sentences analyzable as ' $t$ has location $x_{1}$ or. . or $x_{n}$ (and $R$ is the fusion of $x_{1}, \ldots, x_{n}$ )' and ' $t$ has $z$-spin up or $z$-spin down', respectively. In section 7 , I will consider - and ultimately reject - an alternative interpretation, due to Jessica Wilson, to the effect that an expression like '... has $z$-spin' does not ascribe a disjunctive property of $z$-spin determinates, but rather a nondisjunctive $z$-spin determinable property.

${ }^{17}$ In fact, probability talk is dispensable in the atomic case, as the two parties assign the same interpretation to the primitive physical vocabulary. For sake of uniformity, however, I will deploy a probabilistic paraphrase throughout.
} 
It can be proven that $\mathbf{f}$ preserves coarse-grained content (see sec. A.1 of the appendix). This suffices to show that the classicist can charitably interpret the other party as speaking the truth.

What about the converse, though? It is not as straightforward that the quantum logician can paraphrase the classicist's categorical assertions into her own language. As it turns out, that is possible by semantic ascent to a classical metalanguage. When the classicist asserts the atomic categorical $\mathcal{L}_{C}$-sentence ' $e$ is $z$-spin up', the quantum logician can charitably interpret it as picking out the fact expressed-in- $\mathcal{L}_{Q}$ by ' $e$ is $z$-spin up' is true'. And when the classicist asserts ' $e$ is not $z$-spin up', the other party can charitably interpret that sentence as picking out the fact expressed-in- $\mathcal{L}_{Q}$ by " $e$ is $z$-spin up' is not true', where the latter 'not' is classical. Of course there is nothing problematic with the quantum logician using classical negation, or classical ideology in general, as long as it operates on noncategorical statements such as ' $e$ is $z$-spin up' is true', as opposed to categorical statements such as ' $e$ is $z$-spin'.

With that in mind, let us assume that $\mathcal{L}_{Q}$ is endowed with

i. a classical truth predicate $\mathcal{T}$ such that, for any categorical $\mathcal{L}_{Q}$-sentence $P, \mathcal{T}(P)$ is $\mathcal{L}_{Q^{-}}$true iff $\mathrm{T}_{C}\left([P]_{Q},\left|\psi_{@}\right\rangle\right)$;

ii. a classical negation operator $\sim$, such that for any noncategorical $\mathcal{L}_{Q^{-}}$ sentence $P, \sim P$ is $\mathcal{L}_{Q}$-true iff $P$ is not $\mathcal{L}_{Q}$-true;

iii. a classical conjunction operator \&, such that for any noncategorical $\mathcal{L}_{Q^{-}}$-sentences $S$ and $P, S \& P$ is $\mathcal{L}_{Q^{-}}$-true iff both $S$ and $P$ are $\mathcal{L}_{Q^{-}}$-true.

A paraphrase $\mathbf{g}$ from the categorical fragment of $\mathcal{L}_{C}$ to $\mathcal{L}_{Q}$ is defined as follows:

$\left(\mathrm{At}_{C}\right)$ If $p$ is an atomic categorical sentence of $\mathcal{L}_{C}$, then $\mathbf{g}(p)$ is $\mathcal{T}(p)$.

$(\neg C)$ If $P$ is a categorical sentence of $\mathcal{L}_{C}$, then $\mathbf{g}(\neg P)$ is $\sim \mathbf{g}(P)$.

$\left(\wedge_{C}\right)$ If $S, P$ are categorical sentences of $\mathcal{L}_{C}$, then $\mathbf{g}(S \wedge P)$ is $\mathbf{g}(S) \& \mathbf{g}(P)$.

It can be proven that $\mathbf{g}$ preserves coarse-grained content (see sec. A.2 of the appendix).

Now we know that condition (1) in the definition of verbal disagreement is satisfied, as there exists a paraphrase $\mathbf{g}$ from the categorical fragment of $\mathcal{L}_{C}$ into $\mathcal{L}_{Q}$, and a paraphrase $\mathbf{f}$ from the categorical fragment of $\mathcal{L}_{Q}$ into $\mathcal{L}_{C}$. Condition (2) is also satisfied, for when electron $e$ is in a superposition of $z$-spin up and $z$-spin down, the sentence ' $e$ is not $z$-spin up' is true-in- $\mathcal{L}_{C}$ 
and not true-in- $\mathcal{L}_{Q}$. Therefore, in such circumstances the dispute between classical and quantum logician regarding the truth of ' $e$ is not $z$-spin up' will be verbal in Hirsch's sense. In fact, the two parties can in general disagree merely verbally about the truth value of compound categorical statements involving negation.

But there is more. When $e$ is in a superposition of $z$-spin up and $z$-spin down, neither ' $e$ is $z$-spin up' nor ' $e$ is not $z$-spin up' is true-in- $\mathcal{L}_{Q}$; and since ' $e$ is $z$-spin up' expresses-in- $\mathcal{L}_{Q}$ a fact, and 'not' expresses-in- $\mathcal{L}_{Q}$ the quantum negation operation, it is true-in- $\mathcal{L}_{Q}$ that quantum mechanics entails MI. On the other hand, the classicist's interpretation of $\mathcal{L}_{C}$ is bivalent; so it is not true-in- $\mathcal{L}_{C}$ that quantum mechanics entails MI. Therefore the debate on whether quantum mechanics leads to MI is also verbal. (For a detailed argument, which requires the introduction of an object-language indeterminacy operator, see section A.3 of the appendix.)

In fact, a further consequence can be drawn. Recall that MI is being construed as indeterminacy originating in the world itself, rather than in the way the world is represented. In light of what we just learned, however, the answer to the question 'Is there any indeterminacy independent of our representations?' turns out not to be independent of our representations. We seem to have found evidence supporting a relativistic view to the effect that there is no Archimedean point from which the very question of MI can be answered independently of one's choice of basic concepts (cf. Eklund [19, p. 170]).

\section{$5 \quad$ Naturalness}

\subsection{Substantivity revisited}

In the previous section we reached the twofold conclusion that the question as to whether quantum mechanics entails MI is verbal; and that in general the answer to that question is internal to one's conceptual framework. That conclusion casts a starkly deflationary shadow on the whole first-order debate, as well as on the higher-order debate itself. In this and the following section I will argue that a sufficiently robust form of logical realism - the view that there is one true logic - is able to avoid deflationism about quantum MI. Although I am going to cast logical realism in terms of naturalness, similar arguments could be provided mutatis mutandis on the 
basis of alternative accounts. ${ }^{18}$

One important qualification about the foregoing discussion is that the notion of verbal disagreement was characterized in the style of Hirsch. However, Ted Sider has argued that Hirsch's conditions are necessary, although not jointly sufficient for a dispute to count as verbal. Sider's strategy to evade deflationism in a number of disputes rests on two premises: that (I) the world is endowed with a fundamental structure; and that (II) words get their meanings partly in virtue of what the world's fundamental structure is like.

Premise I is precisified in Lewis [26] by means of the notion of a natural property - roughly, a property which gets referred to in the best description of the actual goings-on. Candidates to being natural in Lewis' sense are firstorder properties of fundamental physics such as mass, charge, etc. Although naturalness is typically treated as primitive, there is a number of key roles that Lewis attributed to the class of natural properties, such as making for qualitative similarity, as well as defining a (minimal) supervenience base. Moreover, naturalness comes in degrees: properties are natural simpliciter when they are perfectly natural; property $\eta$ is at least as natural as property $\theta$ just in case the shortest definition of $\eta$ in purely natural terms is not longer than the shortest definition of $\theta$ in purely natural terms.

Sider [38] has put forward a generalization of Lewis' program by countenancing not only first-order natural properties, but other kinds of items too, such as properties and operations of any order-the semantic values of quantifiers, connectives, and so on. ${ }^{19}$ In particular, Sider thinks that a logical constant such as 'not' has a metaphysically elite meaning, a perfectly natural operation mapping facts to facts - likewise for 'and', 'all', 'some' and other bits of ideology. The one true logic - the one that carves the space of possibility at its joints - is the logic whose constants are interpreted in terms of natural operations only. Since we are only concerned with logical spaces for sentential languages, I will countenance just two sorts of natural items: facts, and operations on facts.

Premise II is part of a metasemantic doctrine known as reference mag-

\footnotetext{
${ }^{18}$ For an assessment of the metaphysical underpinnings of logical realism see McSweeney [33].

${ }^{19}$ In fact, Sider's own formulation of naturalness is quasi-syntactical, insofar as it employs a primitive 'structural' operator applying to linguistic items (predicates, operators etc), rather than worldly items (properties, operations etc). Although Sider's formulation enjoys greater generality, as it does not presuppose any particular metaphysics of higher-order entities, due to my concern with MI I will stick to a worldly formulation in accordance with the Lewisean spirit.
} 
netism (Lewis [26] [27], cf. Merrill [34]), according to which of all candidate meanings for a term, ceteris paribus we should regard the most natural as providing the correct interpretation. If the assignment of semantic values meets such externalist constraints, the substantivity of a dispute is going to be a function of what the world is fundamentally like.

We can now see how Hirsch's characterization can be strengthened by implementing I and II. Let $\mathcal{L}_{C}, \mathcal{L}_{Q}$ be interpreted languages spoken by $C, Q$, respectively, and $\neg p$ a sentence in the common vocabulary. According to Sider, the two parties are said to have a verbal disagreement about the truth of $\neg p$ just in case: ${ }^{20}$

1. there is a two-way paraphrase between $\mathcal{L}_{C}$ and $\mathcal{L}_{Q}$;

2. $\neg p$ is true-in- $\mathcal{L}_{C}$ iff it is not true-in- $\mathcal{L}_{Q} ;$

3. the interpretation-in- $\mathcal{L}_{\mathcal{C}}$ of $\neg$ is just as natural as the interpretationin- $\mathcal{L}_{\mathcal{Q}}$ of $\neg$, and neither interpretation is less natural than any other candidate interpretation of $\neg$.

In particular, if there is a unique perfectly natural negation meaning, disagreement about the truth of $\neg p$ cannot be verbal, since the interpretation of negation will default to that meaning in both languages (provided that the languages coincide in their interpretation of the remaining terms occurring in $p$ ). The above characterization of substantivity applies mutatis mutandis to debates involving sentences governed by other operators.

The import of the new characterization of substantivity for the present debate could not be overstated. For if either complementation or orthocomplementation is natural (and not both), clause (3) of Sider's characterization is not satisfied, and the disagreement between classical and quantum logician concerning the status of MI is going to be substantive. Allow me to elaborate.

\subsection{MI revisited}

In section $2 \mathrm{I}$ defined MI as the phenomenon arising just when some fact is such that neither it nor its negation obtains. However, it should be clear by now that there are multiple ways of carving logical space, depending on a choice of facts and relations over facts. In particular, since there are multiple nonequivalent operations that can play the negation role, the original definition of MI is ambiguous.

\footnotetext{
${ }^{20}$ For an alternative way of implementing I and II in a definition of verbal disagreement, see Torza [41].
} 
Once the Lewis-Sider machinery is in place, however, we can count on a distinguished carving of logical space, which motivates the following revision:

IND*. Metaphysical indeterminacy is said to occur if (i) there is a fact such that neither it nor its negation obtains, and (ii) the relevant negation operation is natural.

Let us take stock. When the MI debate is approached from a metametaphysical perspective, a key question is whether the debate is substantive. On a Hirschean construal of substantivity, the MI debate is verbal, andhorribile dictu - the relevant notion of indeterminacy is not even objective. As we saw, however, such a deflationary moral is not inescapable, for if we buy the Lewis-Sider metaphysical-cum-metasemantic package, the substantivity of the MI debate depends on what candidate meanings are available for 'not'.

In the next section I will delve into the question of whether complementation or orthocomplementation is natural. If either is (but not both), the disagreement between classical and quantum logician is going to be substantive; and which operation is natural will decide, via IND*, whether the quantum world is indeterminate. This development exhibits an interesting interplay between metaphysics and metametaphysics. For we started off by asking the higher-order question whether the first-order debate about quantum indeterminacy is substantive; we learned that the answer to the higherorder question depends on a first-order question, namely how fine-grained the world's structure is; and now we are about to see that the properties of that structure may end up deciding the first-order debate about quantum indeterminacy. Let us take a closer look.

\section{Logical realism}

In this section I turn to two key constraints that have been associated with naturalness, but first a few preliminary notions need to be introduced. As we already know, if $W$ is the set of all possible worlds (states), a logical space $\mathcal{S}$ on $W$ is specified by a set of facts and operations, as well as a relation of obtaining at a world (section 3). For $\mathcal{S}, \mathcal{S}^{\prime}$ logical spaces on $W$, and $P, P^{\prime}$ facts in $\mathcal{S}$ and $\mathcal{S}^{\prime}$, respectively, $P$ is said to be equivalent to $P^{\prime}$ if, for all $w \in W, P$ obtains at $w$ in $\mathcal{S}$ iff $P^{\prime}$ obtains at $w$ in $\mathcal{S}^{\prime}$. (When facts are sets of worlds and the obtaining-at relation is set membership, as is being assumed here, factual equivalence will collapse into set identity.)

Call a logical space $\mathcal{S}$ fundamental when every fact in $\mathcal{S}$ either is natural, or is built up recursively by the application of natural operations to natural 
facts. For example, consider a quantum logical space $\mathcal{Q}$, and suppose that: (i) facts expressed by atomic categorical sentences are natural - these are, intuitively, the facts corresponding to the application of natural properties to quantum particles, such as this photon having that particular position; (ii) logical operations (orthocomplementation, intersection) are natural. Then, $\mathcal{Q}$ will be fundamental provided that every fact in it either is expressed by an atomic categorical sentence, or is built up recursively from the application of quantum-logical operations to facts expressed by atomic categorical sentences. Intuitively, attributing fundamentality to $\mathcal{Q}$ intends to capture the thesis that quantum logic carves the space of possibility at its joints.

I am now going to introduce two constraints on naturalness. The first constraint guarantees that every fact in any space is reducible to some fact in a fundamental space (cf. Sider [38, ch. 7]):

COMPLETEness. Let logical space $\mathcal{S}$ on $W$ be fundamental. Then, for every logical space $\mathcal{S}^{\prime}$ on $W$, every fact in $\mathcal{S}^{\prime}$ is equivalent to some fact in $\mathcal{S}$.

Now, let the Best System be the true axiomatic theory of the world that strikes the best balance with respect to selected epistemic virtues, such as strength and simplicity (Lewis [25] [26]). The second constraint provides an epistemic criterion of naturalness for properties and operations (cf. Sider $[38$, ch. 2.3]):

INDISPENSABILITY. We should regard an operation (or a property) $\eta$ as natural just in case $\eta$ is picked out by a primitive term in the language of the Best System. ${ }^{21}$

This condition tells us that the epistemically most virtuous theory is the place where we should look for evidence of naturalness. Armed with the above two principles, I now return to the case of quantum MI.

All of the best scientific theories currently at our disposal are expressed in the language of classical mathematics. As long as such theories provide a glimpse of what the Best System looks like, we have reason to believe that the Best System too is formulated in classical mathematics. INDISPENSABILITY allows us to infer that the classical operations of negation, disjunction and conjunction are natural - in other words, that the one true logic is classical.

\footnotetext{
${ }^{21}$ This formulation of the criterion presupposes that the Best System is unique, which is highly unlikely. I am setting aside this complication, although the interested reader can see Donaldson [17], Torza [41, sec. 3] for discussion and alternative formulations of INDISPENSABILITY.
} 
It can be concluded that the debate on quantum MI is substantive, and is decided in favor of the classicist. ${ }^{22}$

The above line of thought is defeasible, however. For all we know, the fact that our current epistemically most virtuous theories are phrased against the backdrop of classical logic is a merely historical contingency. In fact, it might well be that the Best System looks very different from any contemporary scientific theory, not only in the way of empirical content but also of logical form - as the advocates of intuitionistic mathematics have long argued, for example. So, the possibility that the one true logic turns out to be nonclassical is not conclusively ruled out.

Be that as it may, there is a second, powerful argument against the thesis that quantum logic carves the space of possibility at its joints. Given the set $W$ of possible states (vectors) in a Hilbert space, let $\mathcal{C}$ be a classical logical space on $W$, and $\mathcal{Q}$ a quantum logical space on $W$. Suppose by way of reductio that $\mathcal{Q}$ is fundamental, and let $P$ be the fact expressed-in- $\mathcal{L}_{C}$ by ' $e$ is not $z$-spin up'. COMPLETENESs entails that $P$ is equivalent to some fact in $\mathcal{Q}$. Since $P$ is not a closed set, however, there is no $P^{\prime}$ in $\mathcal{Q}$ having as members the same vectors as $P$. So, no fact in $\mathcal{Q}$ is equivalent to $P$. Contradiction.

Notice that the quantum logician cannot block the reductio by claiming that since ' $e$ is not $z$-spin up' as uttered by the classicist does not pick out a closed set, it fails to express a fact. For, as seen in section 4 , that sentence can be paraphrased into the quantum logician's language as " $e$ is $z$-spin up' is not true'. The moral is that, although the quantum logician regards $\mathcal{Q}$ as the correct space for interpreting the categorical language on $W$, she must also acknowledge the existence of extra facts on $W$ in order to provide an interpretation for noncategorical statements. Since those extra facts do not correspond to closed sets, the thesis that $\mathcal{Q}$ is fundamental violates COMPLETENESS. Thus, the debate on quantum MI is either verbal, or is won by the classicist.

But all is not lost for the quantum logician. COMPLETEness is a demanding constraint, as it requires that all facts should be reducible one by one to fundamental facts. We could instead adopt a weaker requirement such that the obtaining facts should be collectively reducible to fundamental facts. As it happens, a view in this vicinity has been articulated within the literature on metaphysical ground. For, although it is standard to assume that grounding is a many-one relation, in such a way that a fact is grounded

\footnotetext{
${ }^{22}$ Cf. Sider [38, sec. 9.6.2] for a parallel argument to the effect that classical first-order quantification is natural.
} 
singularly (if at all), it has been argued that we should regard grounding as a many-many relation instead. The latter view has been defended by Dasgupta [16] in the context of a structuralist view of reality. On that view, the ungrounded facts are qualitative (i.e., involving no particular individuals), and the nonqualitative facts are grounded in the qualitative not singularly, but collectively. Structuralist concerns aside, the gist of Dasgupta's point is that in order for the derivative facts to have a ground, it suffices that they be grounded collectively, rather than individually. Once that moral is carried over into naturalness territory, it should be clear that COMPLETENESS is too demanding, and that for the spirit of the principle to be satisfied it suffices that the totality of obtaining facts should be reducible collectively to facts in fundamental logical space. Let us implement this idea.

Call quantum states indistinguishable when they cannot be told apart by any possible experiment. Here is a notion of factual equivalence which generalizes the previous one: for $\mathcal{S}, \mathcal{S}^{\prime}$ logical spaces on $W$, and $\mathbf{P}, \mathbf{P}^{\prime}$ sets of facts in $\mathcal{S}$ and $\mathcal{S}^{\prime}$, respectively, $\mathbf{P}$ is said to be collectively empirically equivalent to $\mathbf{P}^{\prime}$ if, for all $w \in W$, each $P \in \mathbf{P}$ obtains in $\mathcal{S}$ at some state indistinguishable from $w$ iff each $P^{\prime} \in \mathbf{P}^{\prime}$ obtains in $\mathcal{S}^{\prime}$ at some state indistinguishable from $w$. Roughly, sets of facts are collectively empirically equivalent just in case they obtain at the same states up to indistinguishability.

COMPLETENESS can now be replaced with the weaker requirement of collective completeness, which is defined as follows:

C-Completeness. Let logical space $\mathcal{S}$ on $W$ be fundamental. Then, for every logical space $\mathcal{S}^{\prime}$ on $W$, and every $w \in W$, the set of facts obtaining at $w$ in $\mathcal{S}^{\prime}$ is collectively empirically equivalent to some set of facts obtaining at $w$ in $\mathcal{S}$.

Let $\mathcal{C}, \mathcal{Q}$ be classical and quantum logical spaces, respectively, on a set of quantum states $W$. It can be proven that $\mathcal{Q}$ is collectively complete relative to $\mathcal{C}$ (see sec. A.4 of the appendix). Namely, the collection of all classical facts obtaining simpliciter is collectively empirically equivalent to the singleton of the quantum fact which corresponds to the closed set having as members all vectors indistinguishable from $\left|\psi_{@}\right\rangle$. That quantum fact is expressed by an atomic categorical sentence of quantum mechanics.

The above result guarantees that quantum logical space is collectively complete with respect to the classical one. But it is easy to see that the converse result holds, as well. Therefore, considerations of collective completeness, which are stringent but not too stringent, do not tip the balance of the MI debate either way. We can conclude that, if the disagreement is 
substantive, we have not found any conclusive evidence for adjudicating the dispute.

\section{Coda: alternative accounts of MI}

As I mentioned at the end of section 2, the present characterization of MI differs from the two most influential alternatives: the precisificational account of Barnes and Williams [5], and the determinable-based account of Wilson [50]. In this section I explain why I have not adopted either of those readily available views.

According to the Barnes-Williams (BW) approach, MI occurs just when the world is unsettled. The notion of unsettledness is captured by means of the standard distinction between actual world, the concrete object to which we all belong, and representational, aka ersatz worlds. Ersatz worlds can be modeled either linguistically, as maximal consistent sets of sentences in a precise world-making language, or by means of alternative representational machinery. The BW account tells us that the world is metaphysically indeterminate just in case it is indeterminate which ersatz world (ontic precisification) is true.

To start with, the BW account fails to meet the constraint, laid out in section 2, that MI should be defined solely by reference to worldly items, and not by reference to any representational items. Indeed, the BW characterization does not say anything directly about what the world must be like in order for MI to take place, but limits itself to saying what a representation of an indeterminate world must be like. ${ }^{23}$

Second, and most importantly, the BW account is incompatible with quantum MI (Darby [14], Skow [39]). Indeed, the scenario wherein an electron $e$ has $z$-spin up and indeterminate $y$-spin is modeled in terms of two ersatz worlds $w, w^{\prime}$ such that (i) $e$ has $z$-spin up according to both $w$ and $w^{\prime}$; (ii) $e$ has $y$-spin up according to $w$ and $y$-spin down according to $w^{\prime}$. But Heisenberg's uncertainty principle tells us that (ii) is a quantum-mechanical impossibility, and so that there are no such ersatz worlds as $w, w^{\prime}{ }^{24}$

\footnotetext{
${ }^{23}$ Barnes [4] discusses an alternative definition of MI to the effect that, roughly, MI occurs just in case there is a sentence $p$ and a fact $F$ such that $F$ is a truthmaker for $p$, and it is indeterminate whether $F$ exists in actuality. Since the definition quantifies over sentences, it also fails to satisfy my constraint.

${ }^{24} \mathrm{~A}$ variation on the BW approach that restores the possibility of quantum MI by rejecting the precisificational account while retaining the machinery of ersatz worlds is put forward in Torza [40]. For further discussion of the BW approach vis-à-vis quantum MI, see Calosi and Wilson [9]. Darby and Pickup [15] have tried to save the precisificational
} 
A prima facie more promising strategy for modeling quantum indeterminacy is the determinable-based account developed in Wilson [50], and applied to the quantum case in Calosi and Wilson [9] [10]. On this view, MI occurs just when an object has some determinable property $\theta$ (such as $z$-spin) but no unique determinate of $\theta$ (such as up and down).

Three facts about the determinable-based account are relevant here. First of all, the account allows for two ways in which something can be metaphysically indeterminate: gappy indeterminacy, whereby something has a determinable $\theta$ but no determinate of $\theta$; and glutty indeterminacy, whereby something has $\theta$ as well as multiple determinates of $\theta$. Second, Wilson formulates her account of MI against the background of classical bivalent logic, thus presupposing that logical space is classical. Third, the account is defined by appealing to a metaphysical picture, developed in Wilson [49], wherein determinables are irreducible to the respective determinates. The rationale for that assumption is that, contrary to tradition, the determinable-based account requires the instantiation pattern of a determinable to be independent of the instantiation pattern of its determinates. The irreducibility of determinables is made precise in terms of the following condition:

$\mathrm{w}_{1}$. Determinables are not analyzable in terms of their maximally specific determinates. ${ }^{25}$

I take it that a determinable is analyzable in terms of its maximally specific determinates if and only if for something to have the determinable just is for it to have a suitable compound property defined in terms of its maximally specific determinates. Of course there are all sorts of potential strategies for reducing determinables according to that schema. Here is one: for something to have a determinable just is for it to have one of its maximally specific determinates. More precisely, let $\theta$ be a determinable, and $\theta_{1}, \ldots, \theta_{n}$ its maximally specific determinates. Then, $\theta$ is analyzable in terms of $\theta_{1}, \ldots, \theta_{n}$ relative to logical space $\mathcal{X}$ if, for every individual $a,[a \text { is } \theta]_{X}=\left[a\right.$ is $\theta_{1}$ or ... or $a$ is $\left.\theta_{n}\right]_{X}$. Since $\mathrm{w}_{1}$ rules that a determinable is irreducible to its determinates, a fortiori it rules that a determinable is irreducible to a disjunction of its determinates.

view by appealing to situation semantics; but see Corti [13] for a critique of the situationtheoretic approach.

25 "Determinables are not analyzable as disjunctions (or indeed, as any construction of maximal determinates); and [...] it is core to a determinable-based approach to MI that determinables are not so reducible" (Calosi and Wilson [10]). 
By formulating her account against the backdrop of a classical and bivalent logic, Wilson aims to show that making sense of MI does not require any logical revisionism - a goal she shares with Barnes and Williams. I am happy to acknowledge that, other things being equal, preserving classical logic can be regarded as a theoretical virtue. But as discussed in section 3.2 , things are not equal in the context of the orthodox interpretation of quantum mechanics, which offers reasons for identifying facts with closed sets of vectors, and so for preferring quantum to classical logic. In effect, it appears that Calosi and Wilson are not prepared to rule out quantum logic either, since they believe that the quantum-logical approach "is open to a proponent of Determinable-based MI" (Calosi and Wilson [10]). As it turns out, however, quantum logic is incompatible with the determinablebased account, since the latter entails the following condition relative to a quantum logical space $\mathcal{Q}$ :

$\mathrm{W}_{2}$. Some determinables are analyzable in terms of their maximally specific determinates.

In order to see that, let electron $e$ be the only particle in a system associated with quantum logical space $\mathcal{Q}$, and consider the determinableinvolving fact $P:=[e \text { has } z \text {-spin }]_{Q}$. In virtue of being a quantum fact about $e, P$ lives in $\mathcal{Q}$; and in virtue of living in $\mathcal{Q}, P$ is a closed set of vectors. Moreover, $P$ is a necessary fact in $\mathcal{Q}{ }^{26}$ For either $e$ does or does not have a determinate $z$-spin value. If it does then $P$ obtains. This follows from a thesis known as Determinable Inheritance - if $x$ has a determinate of a determinable, then $x$ has the determinable - which is regarded as definitional of the determination relation, and which Calosi and Wilson [10] appear to endorse. And if the $z$-spin value of $e$ is indeterminate, Wilson's definition of MI entails that $P$ obtains, whereas both $[e \text { has } z \text {-spin up }]_{Q}$ and $[e$ has $z$-spin down $]_{Q}$ fail to obtain (or else both obtain).

But here is another fact living in the same logical space: $P^{\prime}:=[e$ has $z$-spin up or $e$ has $z$-spin down $]_{Q}$. Because $[e \text { has } z \text {-spin up }]_{Q}$ and $[e$ has $z$-spin down $]_{Q}$ jointly span $\mathcal{Q}, P^{\prime}$ is a necessary fact in $\mathcal{Q}$ (cf. sec. 3.2). And since quantum facts are sets of vectors, by the extensionality of set membership there is just one necessary fact, hence $P=P^{\prime}$. It follows that in $\mathcal{Q}$ the $z$-spin determinable is analyzable as a disjunction of its maximally specific determinates. This establishes that the the determinable-based account entails $\mathrm{w}_{2}$ relative to quantum logical space $\mathcal{Q}$.

\footnotetext{
${ }^{26}$ That a determinable-involving fact such as $P$ is necessary for the system at hand is not uncontroversial. Indeed, some have argued that, say, $z$-spin talk is simply unintelligible when $e$ 's $z$-spin value is indeterminate (cf. Albert [2, p. 38]).
} 
Since $\mathrm{W}_{2}$ contradicts $\mathrm{w}_{1}$, we must conclude that the metaphysics of irreducible determinables underpinning Wilson's account is incompatible with quantum logic. However, the question whether the logical space defined by a quantum system is classical as opposed to quantum is hardly something that could be settled based on armchair hypotheses regarding the determination relation. Therefore, barring independent reasons for ruling out quantum logic, the determinable-based approach should either be decoupled from the metaphysical assumptions that lead to $\mathrm{w}_{1}$, or abandoned.

\section{Conclusions}

I have tried to shed light on the question whether orthodox quantum mechanics leads to MI by discussing the logical, semantic, and broadly methodological presuppositions of the debate. I have argued that the dispute is verbal if we adopt a Hirschean account of substantivity; but that it need not be so if we embrace Sider's naturalness-based account of substantivity. Given the latter approach, and assuming that the disagreement is substantive, can anything be said in order to tip the balance of the debate between classical and quantum logician either way? Some prima facie reasonable constraints on naturalness entail that the classicist is right, and MI does not arise. As I argued, however, there are reasons to believe that those constraints should be weakened, to the effect that the dispute remains very much open. Finally, I discussed two alternative and influential characterizations of MI, and argued that neither of them is suitable for framing the quantum MI debate. ${ }^{27}$

\section{A Appendix}

\section{A.1 The paraphrase $\mathbf{f}$}

It needs to be proven that the function $\mathbf{f}$ from section 4 , which maps categorical $\mathcal{L}_{Q}$-sentences to $\mathcal{L}_{C}$ sentences, preserves coarse-grained content. The proof is by induction on the syntactical complexity of categorical $\mathcal{L}_{Q^{-}}$

\footnotetext{
${ }^{27}$ I would like to thank Samuel Fletcher, David Glick, Peter Lewis, Elias Okon and the UNAM Metaphysics Seminar for their helpful feedback, as well as audiences at the Quantum Indeterminacy workshop at Dartmouth College, and the 2020 Meeting of the APA Central in Chicago. I am also grateful to two anonymous referees for their thoughtful and generous comments. This work was supported by a PASPA-dgapa sabbatical fellowship which allowed me to spend two semesters at Rutgers University.
} 
sentences. (I will explicitly state the justification of a step only when the step requires appealing to a new principle.)

$\left(\mathrm{At}_{Q}\right)$ We need to show that for any atomic categorical $\mathcal{L}_{Q}$-sentence $p$, and any choice of $\left|\psi_{@}\right\rangle$ :

$$
p \text { is } \mathcal{L}_{Q} \text {-true iff } \operatorname{Pr}\left([p]_{C},\left|\psi_{@}\right\rangle\right)=1 \text { is } \mathcal{L}_{C} \text {-true. }
$$

Proof: $p$ is $\mathcal{L}_{Q}$-true iff $\mathrm{T}_{Q}\left([p]_{Q},\left|\psi_{@}\right\rangle\right)$ (by definition of $\mathcal{L}_{Q}$-truth), iff $\operatorname{Pr}\left([p]_{Q},\left|\psi_{@}\right\rangle\right)=1$ (by CR together with its converse, which is trivial), iff $\operatorname{Pr}\left([p]_{C},\left|\psi_{@}\right\rangle\right)=1$ (since $[p]_{C}=[p]_{Q}$ for atomic $p$ ), iff $\operatorname{Pr}\left([p]_{C},\left|\psi_{@}\right\rangle\right)=1$ is $\mathcal{L}_{C}$-true (since $C$ assigns the correct probabilities to classical facts).

$(\neg Q)$ We need to show that, for any categorical $\mathcal{L}_{Q}$-sentence $P$, and any choice of $\left|\psi_{@}\right\rangle$ :

$\neg P$ is $\mathcal{L}_{Q}$-true iff $\operatorname{Pr}\left([\mathbf{f}(P)]_{C},\left|\psi_{@}\right\rangle\right)=0$ is $\mathcal{L}_{C}$-true.

Proof: $\neg P$ is $\mathcal{L}_{Q}$-true iff $\mathrm{T}_{Q}\left([\neg P]_{Q},\left|\psi_{@}\right\rangle\right)$, iff $\mathrm{T}_{Q}\left(-_{Q}[P]_{Q},\left|\psi_{@}\right\rangle\right)$, iff $\operatorname{Pr}\left(-{ }_{Q}[P]_{Q},\left|\psi_{@}\right\rangle\right)=1$, iff $\operatorname{Pr}\left(-{ }_{Q}[\mathbf{f}(P)]_{C},\left|\psi_{@}\right\rangle\right)=1$ (by inductive hypothesis $)$, iff $\operatorname{Pr}\left([\mathbf{f}(P)]_{C},\left|\psi_{@}\right\rangle\right)=0$, iff $\operatorname{Pr}\left([\mathbf{f}(P)]_{C},\left|\psi_{@}\right\rangle\right)=0$ is $\mathcal{L}_{C}$-true.

$\left(\wedge_{Q}\right)$ We need to show that, for any categorical $\mathcal{L}_{Q}$-sentences $S, P$ and any choice of $\left|\psi_{@}\right\rangle$ :

$S \wedge P$ is $\mathcal{L}_{Q^{-}}$-true iff both $\operatorname{Pr}\left([\mathbf{f}(S)]_{C},\left|\psi_{@}\right\rangle\right)=1$ and $\operatorname{Pr}\left([\mathbf{f}(P)]_{C},\left|\psi_{@}\right\rangle\right)=1$ are $\mathcal{L}_{C}$-true.

Proof: $S \wedge P$ is $\mathcal{L}_{Q}$-true iff $\mathrm{T}_{Q}\left([S \wedge P]_{Q},\left|\psi_{@}\right\rangle\right)$, iff $\mathrm{T}_{Q}\left([S]_{Q} \sqcap_{Q}[P]_{Q},\left|\psi_{@}\right\rangle\right)$, iff $\operatorname{Pr}\left([S]_{Q} \sqcap_{Q}[P]_{Q},\left|\psi_{@}\right\rangle\right)=1$, iff $\operatorname{Pr}\left([\mathbf{f}(S)]_{C} \sqcap_{Q}[\mathbf{f}(P)]_{C},\left|\psi_{@}\right\rangle\right)=1$ (by inductive hypothesis), iff $\operatorname{Pr}\left([\mathbf{f}(S)]_{C} \sqcap_{C}[\mathbf{f}(P)]_{C},\left|\psi_{@}\right\rangle\right)=1$ (because $\left.\Pi_{C}=\Pi_{Q}\right)$, iff both $\operatorname{Pr}\left([\mathbf{f}(S)]_{C},\left|\psi_{@}\right\rangle\right)=1$ and $\operatorname{Pr}\left([\mathbf{f}(P)]_{C},\left|\psi_{@}\right\rangle\right)=1$, iff both $\operatorname{Pr}\left([\mathbf{f}(S)]_{C},\left|\psi_{@}\right\rangle\right)=1$ and $\operatorname{Pr}\left([\mathbf{f}(P)]_{C},\left|\psi_{@}\right\rangle\right)=1$ are $\mathcal{L}_{C}$-true.

\section{A.2 The paraphrase $\mathrm{g}$}

It needs to be proven that the function $\mathbf{g}$ from section 4 , which maps categorical $\mathcal{L}_{C}$-sentences to $\mathcal{L}_{Q}$ sentences, preserves coarse-grained content. The proof is by induction on the syntactical complexity of categorical $\mathcal{L}_{C^{-}}$ sentences. (Once again, I will explicitly state the justification of a step only when a new principle is appealed to.) 
$\left(\mathrm{At}_{C}\right)$ We need to show that for any atomic categorical $\mathcal{L}_{C}$-sentence $p$, and any choice of $\left|\psi_{@}\right\rangle$ :

$$
p \text { is } \mathcal{L}_{C} \text {-true iff } \mathcal{T}(p) \text { is } \mathcal{L}_{Q} \text {-true. }
$$

Proof: $p$ is $\mathcal{L}_{C}$-true iff $\mathrm{T}_{C}\left([p]_{C},\left|\psi_{@}\right\rangle\right)$ (by definition of $\mathcal{L}_{C}$-truth), iff $\mathrm{T}_{C}\left([p]_{Q},\left|\psi_{@}\right\rangle\right)$ (since $[p]_{C}=[p]_{Q}$ for atomic $\left.p\right)$, iff $\mathcal{T}(p)$ is $\mathcal{L}_{Q}$-true (by definition of $\mathcal{T})$.

$(\neg C)$ We need to show that, for any categorical $\mathcal{L}_{C}$-sentence $P$, and any choice of $\left|\psi_{@}\right\rangle$ :

$$
\neg P \text { is } \mathcal{L}_{C} \text {-true iff } \sim \mathbf{g}(P) \text { is } \mathcal{L}_{Q} \text {-true. }
$$

Proof: $\neg P$ is $\mathcal{L}_{C}$-true iff $\mathrm{T}_{C}\left([\neg P]_{C},\left|\psi_{@}\right\rangle\right)$, iff $\mathrm{T}_{C}\left(-_{C}[P]_{C},\left|\psi_{@}\right\rangle\right)$, iff not $\mathrm{T}_{C}\left([P]_{C},\left|\psi_{@}\right\rangle\right)$, iff not $\mathrm{T}_{C}\left([\mathbf{g}(P)]_{Q},\left|\psi_{@}\right\rangle\right)$ (by inductive hypothesis), iff $\mathbf{g}(P)$ is not $\mathcal{L}_{Q^{-}}$true, iff $\sim \mathbf{g}(P)$ is $\mathcal{L}_{Q^{-}}$true (by definition of $\sim$ ).

$\left(\wedge_{C}\right)$ We need to show that, for any categorical $\mathcal{L}_{C}$-sentences $S, P$ and any choice of $\left|\psi_{@}\right\rangle$ :

$$
S \wedge P \text { is } \mathcal{L}_{C} \text {-true iff } \mathbf{g}(S) \& \mathbf{g}(P) \text { is } \mathcal{L}_{Q^{-}} \text {true. }
$$

Proof: analogous to the case of $(\neg C)$.

\section{A.3 Verbal disagreement about MI}

We want to show that the dispute between classicists $\left(\mathcal{L}_{C}\right.$-speakers $)$ and quantum logicians $\left(\mathcal{L}_{Q}\right.$-speakers $)$ about whether quantum mechanics leads to MI is verbal in Hirsch's sense (sec. 4). A rigorous proof that the dispute is verbal will require (i) introducing an indeterminacy operator in both object languages; (ii) extending the paraphrase schemes $\mathbf{f}, \mathbf{g}$ in order to account for statements of indeterminacy; (iii) showing that, for some categorical sentence $p$, 'it is metaphysically indeterminate whether $p$ ' is true-in- $\mathcal{L}_{C}$ iff it is not true-in- $\mathcal{L}_{Q}$.

Let us start by expanding both $\mathcal{L}_{C}$ and $\mathcal{L}_{Q}$ with a sentential operator $\nabla$ with the intended meaning 'it is metaphysically indeterminate whether' as defined by IND. We then extend $\mathbf{f}$ to a mapping $\mathbf{f}^{*}$ from categorical $\mathcal{L}_{Q^{-}}$-sentences to $\mathcal{L}_{C}$ sentences such that:

$\left(\nabla_{Q}\right)$ If $P$ is a categorical sentence of $\mathcal{L}_{Q}$, then $\mathbf{f}^{*}(\nabla P)$ is $\neg \mathbf{P r}\left(\left[\mathbf{f}^{*}(P)\right]_{C},\left|\psi_{@}\right\rangle\right)$

$$
=1 \wedge \neg \operatorname{Pr}\left(\left[\mathbf{f}^{*}(P)\right]_{C},\left|\psi_{@}\right\rangle\right)=0
$$


whereas $\mathbf{f}^{*}(P)=\mathbf{f}(P)$ if $P$ is atomic, negated, or conjunctive. We need to show that for any categorical $\mathcal{L}_{Q}$-sentence $P$, and any choice of $\left|\psi_{@}\right\rangle$ :

$\nabla P$ is $\mathcal{L}_{Q}$-true iff $\neg \operatorname{Pr}\left(\left[\mathbf{f}^{*}(P)\right]_{C},\left|\psi_{@}\right\rangle\right)=1 \wedge \neg \operatorname{Pr}\left(\left[\mathbf{f}^{*}(P)\right]_{C},\left|\psi_{@}\right\rangle\right)=$ 0 is $\mathcal{L}_{C}$-true.

Proof: $\nabla P$ is $\mathcal{L}_{Q}$-true iff not $\mathrm{T}_{Q}\left([P]_{Q},\left|\psi_{@}\right\rangle\right)$ and not $\mathrm{T}_{Q}\left([\neg P]_{Q},\left|\psi_{@}\right\rangle\right)$, iff not $\mathrm{T}_{Q}\left([P]_{Q},\left|\psi_{@}\right\rangle\right)$ and not $\mathrm{T}_{Q}\left(-{ }_{Q}[P]_{Q},\left|\psi_{@}\right\rangle\right)$, iff not $\operatorname{Pr}\left([P]_{Q},\left|\psi_{@}\right\rangle\right)=1$ and not $\operatorname{Pr}\left({ }_{Q}[P]_{Q},\left|\psi_{@}\right\rangle\right)=1$, iff not $\operatorname{Pr}\left(\left[\mathbf{f}^{*}(P)\right]_{C},\left|\psi_{@}\right\rangle\right)=1$ and not $\operatorname{Pr}\left(-_{Q}\left[\mathbf{f}^{*}(P)\right]_{C},\left|\psi_{@}\right\rangle\right)=1$ (by inductive hypothesis), iff not $\operatorname{Pr}\left(\left[\mathbf{f}^{*}(P)\right]_{C},\left|\psi_{@}\right\rangle\right)$ $=1$ and not $\operatorname{Pr}\left(\left[\mathbf{f}^{*}(P)\right]_{C},\left|\psi_{@}\right\rangle\right)=0$, iff $\neg \operatorname{Pr}\left(\left[\mathbf{f}^{*}(P)\right]_{C},\left|\psi_{@}\right\rangle\right)=1 \wedge \neg \operatorname{Pr}\left(\left[\mathbf{f}^{*}(P)\right]_{C},\left|\psi_{@}\right\rangle\right)=$ 0 is $\mathcal{L}_{C}$-true.

We now extend $\mathbf{g}$ to a mapping $\mathbf{g}^{*}$ from categorical $\mathcal{L}_{C}$-sentences to $\mathcal{L}_{Q}$ sentences such that

$\left(\nabla_{C}\right)$ If $P$ is a categorical sentence of $\mathcal{L}_{C}$, then $\mathbf{g}^{*}(\nabla P)$ is $0=1$

whereas $\mathbf{g}^{*}(P)=\mathbf{g}(P)$ if $P$ is atomic, negated, or conjunctive. We need to show that for any categorical $\mathcal{L}_{C}$-sentence $P$, and any choice of $\left|\psi_{@}\right\rangle$ :

$\nabla P$ is $\mathcal{L}_{C}$-true iff $0=1$ is $\mathcal{L}_{Q}$-true.

Since $\mathcal{L}_{C}$ is bivalent, the proof is trivial. This concludes the proof that there is a two-way paraphrase between expanded $\mathcal{L}_{Q}$ and expanded $\mathcal{L}_{C}$.

The final step is to find a categorical sentence $p$ in the common fragment of $\mathcal{L}_{C}$ and $\mathcal{L}_{Q}$ such that $\nabla p$ is true-in- $\mathcal{L}_{Q}$ and not true-in- $\mathcal{L}_{C}$. Relative to a single-electron system in a superposition of $z$-spin up and $z$-spin down, one such sentence is ' $e$ is $z$-spin up.' This suffices to show that the disagreement between $C$ and $Q$ concerning quantum MI is verbal in Hirsch's sense.

\section{A.4 Collective completeness of $\mathcal{Q}$ relative to $\mathcal{C}$}

Let $\mathcal{C}, \mathcal{Q}$ be classical and quantum logical spaces, respectively, on a set of quantum states $W$. We need to show that $\mathcal{Q}$ is collectively complete relative to $\mathcal{C}$, which is to say, that the collection of all classical facts obtaining at $\left|\psi_{@}\right\rangle$ is collectively empirically equivalent to the singleton of the quantum fact which corresponds to the closed set having as members all vectors indistinguishable from $\left|\psi_{@}\right\rangle$. The proof is as follows.

In quantum mechanics, states differing by a scalar verify the same experimental facts. Therefore, quantum states are indistinguishable just in case they differ by a scalar. Let $\mathbf{P}$ be the set of facts obtaining at $\left|\psi_{@}\right\rangle$ in $\mathcal{C}$. Consider now the set of facts $\mathbf{Q}=\{Q\}$ in $\mathcal{Q}$, where $Q$ is the set 
of unitary vectors indistinguishable from $\left|\psi_{@}\right\rangle$. If $Q$ obtains at some state indistinguishable from a state $|\psi\rangle$, then $\left|\psi_{@}\right\rangle$ and $|\psi\rangle$ are indistinguishable. Therefore, every $P \in \mathbf{P}$ obtains at some state, namely $\left|\psi_{@}\right\rangle$, which is indistinguishable from $|\psi\rangle$. Conversely, let every $P \in \mathbf{P}$ obtain at some state indistinguishable from a state $|\psi\rangle$. Since $Q \in \mathbf{P},\left|\psi_{@}\right\rangle$ and $|\psi\rangle$ are indistinguishable. Therefore, $Q$ obtains at some state, namely $\left|\psi_{@}\right\rangle$, which is indistinguishable from $|\psi\rangle$. QED.

\section{References}

[1] Akiba, Ken (2004). Vagueness in the world. Nô̂s 38: 407-29.

[2] Albert, David Z. (2009). Quantum mechanics and experience. Cambridge: Harvard University Press.

[3] Bacciagaluppi, Guido (2009). Is logic empirical? In Engesser, K., Gabbay, D. and Lehmann, D. (eds). Handbook of quantum logic and quantum structures: Quantum logic. Elsevier: 49-78.

[4] Barnes, Elizabeth (2010). Ontic Vagueness: A Guide for the Perplexed. Noûs 44 (4): 601-627.

[5] Barnes, Elizabeth and Williams, J. Robert G. (2011). A Theory of Metaphysical Indeterminacy. In Zimmerman, D. and Bennett, K. (eds) Oxford Studies in Metaphysics vol. 6: 103-48. Oxford: Oxford University Press.

[6] Birkhoff, Garrett, and von Neumann, John. (1936). The logic of quantum mechanics. The Annals of Mathematics, 37(4): 823-843.

[7] Bokulich, Alisa (2014). Metaphysical Indeterminacy, Properties, and Quantum Theory. Res Philosophica 91 (3):449-475.

[8] Calosi, Claudio and Mariani, Cristian (2021). Quantum indeterminacy. Philosophy Compass.

[9] Calosi, Claudio and Wilson, Jessica M. (2019). Quantum Metaphysical Indeterminacy. Philosophical Studies 176: 2599-2627.

[10] Calosi, Claudio and Wilson, Jessica M. (2021). Quantum Indeterminacy and the Double-Slit Experiment. Philosophical Studies. 
[11] Cameron, Ross (2011). Truthmaking for presentists. In Zimmerman, D. and Bennett, K. (eds) Oxford Studies in Metaphysics vol. 6: 55-100. Oxford: Oxford University Press.

[12] Chen, Eddy Keming (forthcoming). Fundamental Nomic Vagueness. Philosophical Review.

[13] Corti, Alberto (2021). Yet again, quantum indeterminacy is not worldly indecision. Synthese.

[14] Darby, George (2010). Quantum Mechanics and Metaphysical Indeterminacy. Australasian Journal of Philosophy 88 (2): 227-245.

[15] Darby, George and Pickup, Martin (2021). Modelling Deep Indeterminacy. Synthese 198: 1685-1710.

[16] Dasgupta, Shamik (2014). On the Plurality of Grounds. Philosophers' Imprint 14.

[17] Donaldson, Tom (2014). Reading the Book of the World. Philosophical Studies 172 (4): 1051-77.

[18] Einstein, Albert, Podolsky, B., and Rosen, N. (1935). Can quantummechanical description of physical reality be considered complete? Physical review, 47(10): 777.

[19] Eklund, Matti (2011). Being Metaphysically Unsettled: Barnes and Williams on Metaphysical Indeterminacy and Vagueness. In Bennett, K. and Zimmerman, D. (eds) Oxford Studies in Metaphysics vol. 6. Oxford University Press.

[20] Fine, Kit (1975). Vagueness, truth, and logic. Synthese 30(3-4): 265300.

[21] Glick, David (2017). Against Quantum Indeterminacy. Thought: A Journal of Philosophy 6 (3): 204-13.

[22] Hirsch, Eli (2009). Ontology and Alternative Languages. In: Chalmers, D., Manley, D., and Wasserman, R. (eds). Metametaphysics. Oxford University Press.

[23] Hirsch, Eli (2011). Quantifier Variance and Realism: Essays in Metaontology. Oxford University Press, USA. 
[24] Gilton, Marian J. R. (2016). Whence the eigenstate-eigenvalue link? Studies in History and Philosophy of Science Part B: Studies in History and Philosophy of Modern Physics 55: 92-100.

[25] Lewis, David K. (1973). Counterfactuals. Blackwell.

[26] Lewis, David K. (1983). New work for a theory of universals. Australasian Journal of Philosophy 61 (4): 343-377.

[27] Lewis, David K. (1984). Putnam's paradox. Australasian Journal of Philosophy 62 (3): 221-236.

[28] Lewis, David K. (1986). On the Plurality of Worlds. Wiley-Blackwell.

[29] Lewis, Peter J. (2016). Quantum Ontology: A Guide to the Metaphysics of Quantum Mechanics. Oxford University Press USA.

[30] Lowe, E. J. (1994). Vague Identity and Quantum Indeterminacy. Analysis 54(2): 110-14.

[31] Lowe, E. J. (1999). Vague Identity and Quantum Indeterminacy: Further Reflections. Analysis 59(264): 328-30.

[32] Maudlin, Tim (2014). What Bell did. Journal of Physics A: Mathematical and Theoretical, 47(42): 424010.

[33] McSweeney, Michaela Markham (2019). Logical Realism and the Metaphysics of Logic. Philosophy Compass 14 (1): e12563.

[34] Merrill, G. H. (1980). The model-theoretic argument against realism. Philosophy of Science 47 (1): 69-81.

[35] Noonan, Harold W. (1995). E. J. Lowe on Vague Identity and Quantum Indeterminacy. Analysis 55 (1): 14-19.

[36] Putnam, Hilary (1968). Is logic empirical? In Cohen, R. and Wartofsky, M. (eds). Boston Studies in the Philosophy of Science, vol. 5 Dordrecht: Reidel: 216-241.

[37] Rayo, Agustín (2017). The World is the Totality of Facts, Not of Things. Philosophical Issues 27 (1): 250-278.

[38] Sider, Theodore (2011). Writing the Book of the World. Oxford University Press. 
[39] Skow, Bradford (2010). Deep metaphysical indeterminacy. Philosophical Quarterly 60 (241): 851-58.

[40] Torza, Alessandro (2020). Quantum metaphysical indeterminacy and worldly incompleteness. Synthese 197: 4251-4264.

[41] Torza, Alessandro (2020). Structural Indeterminacy. Philosophy and Phenomenological Research 101(2): 365-382.

[42] Turner, Jason (2016). The Facts in Logical Space: A Tractarian Ontology. Oxford University Press.

[43] Wallace, David (2012). Decoherence and its role in the modern measurement problem. Philosophical Transactions of the Royal Society A: Mathematical, Physical and Engineering Sciences, 370(1975): 45764593.

[44] Wallace, David (2019). What is Orthodox Quantum Mechanics? In Alberto Cordero (ed.), Philosophers Look at Quantum Mechanics. Springer.

[45] Williams, J. Robert G. (2008). Ontic vagueness and metaphysical indeterminacy. Philosophy Compass 3 (4): 763-88.

[46] Williams, J. Robert G. (2008). Multiple actualities and ontically vague identity. The Philosophical Quarterly 58(230): 134-54.

[47] Williamson, Tim (1994). Vagueness. London: Routledge.

[48] Williamson, Tim (2003). Vagueness in reality. In Loux, M. and Zimmerman, D. (eds.), The Oxford Handbook of Metaphysics. Oxford University Press.

[49] Wilson, Jessica M. (2012). Fundamental determinables. Philosophers' Imprint 12.

[50] Wilson, Jessica M. (2013). A Determinable-Based Account of Metaphysical Indeterminacy. Inquiry 56 (4): 359-85. 